\title{
The probiotic Escherichia coli strain Nissle 1917 (EcN) stops acute diarrhoea in infants and toddlers
}

\author{
Jobst Henker • Martin Laass • Boris M. Blokhin • \\ Yuriy K. Bolbot • Vitaliy G. Maydannik • \\ Martina Elze • Corinna Wolff • Jürgen Schulze
}

Received: 26 October 2006 / Revised: 20 December 2006 / Accepted: 29 December 2006 / Published online: 8 February 2007

(C) Springer-Verlag 2007

\begin{abstract}
In most cases, acute diarrhoea will become selflimiting during the first few days after onset. For young children, however, health risks may develop when the disease lasts longer than 3 days. The purpose of the present
\end{abstract}

Electronic supplementary material The online version of this article (doi:10.1007/s00431-007-0419-x) contains supplementary material, which is available to authorized users.

Supported by ARDEYPHARM through the provision of verum and placebo medication and reimbursement of study-related expenditure. The authors would like to thank all hospital staff members, clinical monitors and parents for contributing to the work achieved.

J. Henker $(\bowtie) \cdot$ M. Laass

Department of Paediatrics,

University Hospital Carl-Gustav-Carus,

Fetscherstrasse 74,

01307 Dresden, Germany

e-mail: Jobst.Henker@uniklinikum-dresden.de

B. M. Blokhin

Outpatient Paediatrics, Russian State Medical University,

Moscow, Russia

\section{Y. K. Bolbot}

Paediatrics No. 2, Dnepropetrovsk State Medical Academy,

Dnepropetrovsk, Ukraine

\section{G. Maydannik}

Paediatrics No. 4, City Paediatric Clinical Hospital No. 6, National Medical University,

O. O. Bogomolets,

Kyiv, Ukraine

\section{Elze}

ClinResearch, Institute for Monitoring, Data Management,

Biometrics and Medical Writing,

Cologne, Germany

C. Wolff · J. Schulze

Ardeypharm,

Herdecke, Germany trial was to determine whether the stool frequency of infants and toddlers suffering from acute diarrhoea could be normalised more quickly by administering the probiotic Escherichia coli Nissle $1917(\mathrm{EcN})$ solution than by administering a placebo. The safety of $\mathrm{EcN}$ were also assessed. A total of 113 children (aged 2-47 months) with acute diarrhoea ( $>$ three watery or loose stools in $24 \mathrm{~h}$ ) were randomised to either a group receiving the probiotic $\mathrm{EcN}$ suspension $(n=55)$ or a group receiving the placebo suspension $(n=58)$ in a confirmative, double-blind clinical trial. Depending on the age of patients, $1-3 \mathrm{ml}$ per day of verum suspension $\left(10^{8}\right.$ viable $\mathrm{EcN}$ cells per millilitre) or placebo were administered orally. The causes of the diarrhoea were viral rather than bacterial, but they were mainly unspecific infections. The median onset of treatment response (reduction of daily stool frequency to $\leq$ three watery or loose stools over at least 2 consecutive days) occurred more rapidly in the children receiving the $\mathrm{EcN}$ solution (2.5 days) than in those receiving the placebo (4.8 days), a significant difference ( 2.3 days; $p=0.0007$ ). The number of patients showing a response was clearly higher $(p<0.0001)$ in the $\mathrm{EcN}$ group $(52 / 55 ; 94.5 \%)$ than in the placebo group $(39 / 58 ; 67.2 \%)$. EcN was found to be safe and well-tolerated, and it showed a significant superiority compared to the placebo in the treatment of acute diarrhoea in infants and toddlers.

Keywords Acute diarrhoea $\cdot$ EcN $\cdot$ Escherichia coli Nissle $1917 \cdot$ Infants $\cdot$ Probiotic $\cdot$ Toddlers
Abbreviations
ADDPLAN Adaptive Designs - Plans and Analyses
AE adverse event
BMI body mass index $\left(\mathrm{kg} / \mathrm{m}^{2}\right)$
CI confidence interval 


$\begin{array}{ll}\text { CPMP } & \text { Committee for Proprietary Medicinal Products } \\ \text { EcN } & \text { Escherichia coli strain Nissle 1917 } \\ \text { EMEA } & \text { European Medicines Agency } \\ \text { ICH } & \text { International Conference on Harmonization } \\ \text { IEC } & \text { Independent Ethics Committee } \\ \text { IDMC } & \text { Independent Data Monitoring Committee } \\ \text { ITT } & \text { intention to treat } \\ \text { PP } & \text { per protocol }\end{array}$

\section{Introduction}

Probiotics are non-pathogenic microorganisms - mostly of human origin - which confer health benefits to the host when administered in adequate amounts. They are considered to be a safe and effective part of the first-line therapy for acute diarrhoea in children and adults [12]. In addition, probiotics are able to prevent or improve not only gastrointestinal diseases such as inflammatory bowel disease, irritable bowel syndrome, infectious gastroenteritis or diverticular disease of the colon, but also to act in the prevention of allergic diseases.

Various probiotics are commercially available in, for example, Europe, the USA and Japan where they are marketed as functional foods or probiotic drugs. To date, lactobacilli, bifidobacteria and Saccharomyces boulardii are the most commonly marketed probiotic active substances. Certain strains of Escherichia coli are also available in some European countries, the best known example of which is E. coli strain Nissle 1917 (EcN).

$\mathrm{EcN}$ is marketed as a probiotic drug in two galenic presentations for oral use: enteric-coated capsules and a suspension in which $1 \mathrm{ml}$ contains $10^{8}$ viable EcN cells. While capsules are mostly used in adults (e.g. Kruis et al. [8]), the use of the suspension form is the most reasonable form in neonates, infants and toddlers.

The purpose of the present trial was to examine the efficacy and safety of an $\mathrm{EcN}$ suspension administered to infants and toddlers suffering from acute diarrhoea of different causes in terms of normalising the stool frequency.

\section{Materials and methods}

Infants and toddlers treated for acute diarrhoea in the paediatric outpatient wards of 11 centres between February and April 2005 were eligible for enrollment in this study. This was a multicentre, prospective, confirmative, randomised, double-blind, placebo-controlled, parallel group clinical trial of phase III. It was carried out in accordance with the requirements of Good Clinical Practice and the Revised Declaration of Helsinki. The study was approved by the

Independent Ethics Committee (IEC) of the Federal Agency of Drugs Quality Control, Moscow, Russia, and by the IEC of the State Enterprise Centre of Immunobiological Medicines at the Ministry of Health of Ukraine.

Acute diarrhoea was defined as more than three wateryto-loose stools per day from an acute episode of non-bloody diarrhoea which did not persist longer than 3 successive days. For reasons of comparability, one of the exclusion criteria was a higher grade of dehydration (loss of body weight $>5 \%$; hydration status was surveyed, rehydration was not implemented in low-grade dehydration); the most important inclusion and exclusion criteria are listed in Table 1. The participant was assessed until ascertainment of response, 10 days at maximum. An overview of the study design is presented in Fig. 1. A stool sample was taken at both the beginning and end of the study and checked for the presence of the following pathogens: Salmonella, Campylobacter, Yersinia, E. coli (ETEC, EPEC, EIEC, EHEC), Shigella, Entamoeba histolytica, Cryptosporidium parvum and Rota-, Adeno- and Noroviruses.

The parents were asked to maintain a daily record (diary) containing information on the number of stools, stool consistency, admixtures of blood or mucus, frequency of vomiting, abdominal pain and cramps and fluid intake as well as concomitant medication and general state of health. An assessment of general health was also documented during each control visit by the investigator and parents.

The randomisation schedule was generated by means of SAS, ver. 9.1 (SAS Institute, Cary, N.C.) based on seed values dependent on a random number generator. The method of randomly permuted blocks was used (block size: 4 ).

\section{Study medication}

The drug being studied (verum) is a commercially available suspension for oral use that contains non-pathogenic E. coli strain Nissle 1917 (Mutaflor suspension; Ardeypharm, Herdecke, Germany, with $10^{8}$ viable microorganisms per millilitre). As placebo, we administered an identical preparation consisting of a suspension devoid of the active substance. In accordance with good clinical practice (GCP), identical containers were used in order to guarantee a concealed random allocation both to the parents and the

Infants $<1$ year

Toddlers $\geq 1$ to $\leq 3$ years

Toddlers $>3$ to $<4$ years

study personnel involved. Depending on the age, daily doses of the study medication ( $\mathrm{EcN}$ or placebo) were: 
Table 1 Inclusion and exclusion criteria

Inclusion criteria

Age $<4$ years at the time of enrolment

More than three watery or loose non-bloody stools in a 24 -h period

that had not persisted for more than three consecutive days

Signed informed consent by the parents
Exclusion criteria

Dehydration ( $>5 \%$ loss of body weight)

Participation in another clinical trial

Intake of $\mathrm{EcN}$ within the past 3 months prior to enrolment

Intake of food supplements or drugs which contain living microorganisms or their metabolic products or components within 7 days prior to enrolment or during the trial

Other antidiarrhoeal drugs

Antibiotics

Breast-feeding, Premature birth

Severe or chronic disease of the bowel or severe concomitant diseases
The parents received a diary in which the intake of the trial medication was documented. The investigator checked the entries for completeness and plausibility. The compliance was also evaluated by comparing the amount of trial medication handed out with that returns.

\section{Outcome}

The primary effect criterion was the time to response. Treatment response was defined as a reduction in stool frequency to $\leq$ three watery or loose stools in $24 \mathrm{~h}$ over a period of at least 2 consecutive days. Secondary effect criteria included the response rate, stool consistency, abdominal pain and cramps, body temperature, frequency of vomiting, occurrence of adverse events and tolerance to the study medication.

\section{Statistical analysis}

The study was conducted according to a three-stage group sequential test design (O'Brien/Fleming type) with possible sample size adaptation after the two planned interim analyses [9]. A time-to-response analysis was performed (Kaplan-Meier method; log-rank test to test the superiority of EcN compared to placebo; overall type-I error rate $\alpha=0.025$; one-sided). The response rates were also computed and compared between treatment groups by means of Fisher's exact test (one-sided; exploratory). The intentionto-treat (ITT) data set included all randomised patients who took at least one dose of study medication (primary analysis), whereas patients with major protocol violations were excluded from the per-protocol (PP) analysis. The analysis sets were defined in a blind review of the data. The sample size was estimated prospectively using ADDPLAN ver. 3.0. An independent data monitoring committee (IDMC) was responsible for reviewing the results of the interim analyses and giving recommendations. Two interim analyses were performed, resulting in continuation of the study with the pre-planned sample sizes.

\section{Results}

\section{Baseline data}

A total of 113 infants and toddlers between 2 and 47 months of age with acute diarrhoea were admitted to the trial. All patients were Caucasian. The patients were randomly allocated to either the $\mathrm{EcN}$ group (55 patients) or the placebo group (58 patients) (Fig. 2). No relevant differences between the groups were observed in terms of gender, age, height, weight and BMI of the patients (Table 2). The vast majority of patients had an average body development and a good nutritional status, but reduced appetite was reported. There were also no differences in systolic and diastolic blood pressure, heart rate and body temperature between the two treatment groups at baseline.
Fig. 1 Study design. *Final visit Duration of treatment, which was until ascertainment of response, 10 days at maximum

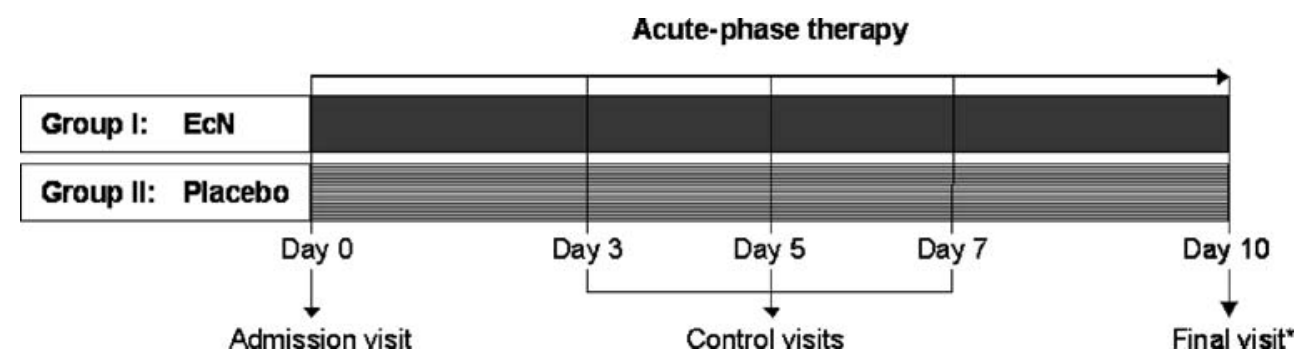




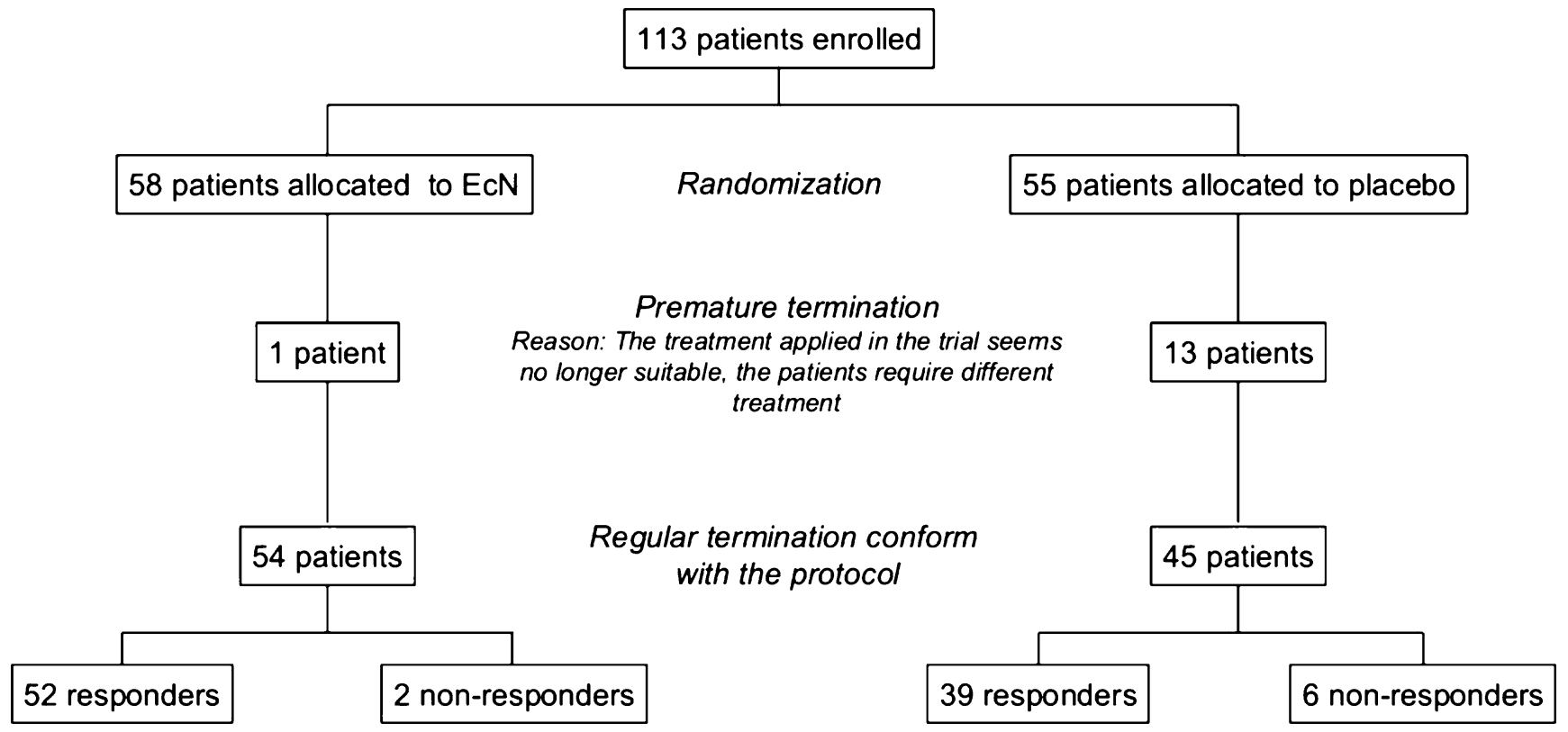

Fig. 2 Diagram of participant flow

There was no difference in the duration of the current acute diarrhoea episode between patients in the $\mathrm{EcN}$ group and placebo group (EcN: $1.4 \pm 0.6$ days, mean: 1.0 days; placebo: $1.6 \pm 0.6$ days, mean: 2.0 days). The number of infections during the past 12 months was $\leq$ five in $55 / 55$ patients $(100 \%)$ of the $\mathrm{EcN}$ group and $56 / 58$ patients $(96.6 \%)$ of the placebo group. Infections as a cause of the acute diarrhoea were more often viral than bacterial. However, unspecific infections were the most common (Table 2). The number and proportion of patients with pathogenic microorganisms were slightly higher in the $\mathrm{EcN}$ group (27/55 patients) than in the placebo group (21/58 patients) at baseline. This difference was not statistically significant.
Data analyses

All efficacy analyses were originally designed to be performed on both the ITT and PP data sets. However, as the ITT and PP data sets were identical in this study $(n=113$ patients), only the ITT data are evaluated here. All safety analyses were performed on the ITT data set.

Primary objective

The median time to response was 2.5 days in the $\mathrm{EcN}$ group and 4.8 days in the placebo group, i.e. treatment with $\mathrm{EcN}$ shortened the duration of diarrhoea by 2.3 days. Statistical testing revealed that the $\mathrm{EcN}$ treatment was

Table 2 Baseline data for the two treatment groups

\begin{tabular}{|c|c|c|c|}
\hline & & $\mathrm{EcN}(n=55)$ & Placebo $(n=58)$ \\
\hline Male gender & & $32(58.2 \%)$ & $32(55.2 \%)$ \\
\hline Age (median) & & 21 months & 23 months \\
\hline Height (median) & & $83 \mathrm{~cm}$ & $83 \mathrm{~cm}$ \\
\hline Weight (median) & & $12.7 \mathrm{~kg}$ & $12.6 \mathrm{~kg}$ \\
\hline BMI (median) & & $17.4 \mathrm{~kg} / \mathrm{m}^{2}$ & $17.2 \mathrm{~kg} / \mathrm{m}^{2}$ \\
\hline Mean duration of diarrhoea & & 1.4 days & 1.6 days \\
\hline Mean stool frequency & & 5.0 per day & 5.1 per day \\
\hline \multirow[t]{5}{*}{ Possible causes for the current acute diarrhoea episode } & Previous antibiotic treatment & $2(3.6 \%)$ & $4(6.9 \%)$ \\
\hline & Virus infections & $16(29.1 \%)$ & $19(32.8 \%)$ \\
\hline & Bacterial infections & $9(16.4 \%)$ & $4(6.8 \%)$ \\
\hline & Unspecified infections & $25(45.5 \%)$ & $29(50.0 \%)$ \\
\hline & Other causes & $3(5.5 \%)$ & $2(3.4 \%)$ \\
\hline
\end{tabular}


Fig. 3 Time-to-response curves: Kaplan-Meier analysis (ITT analysis)

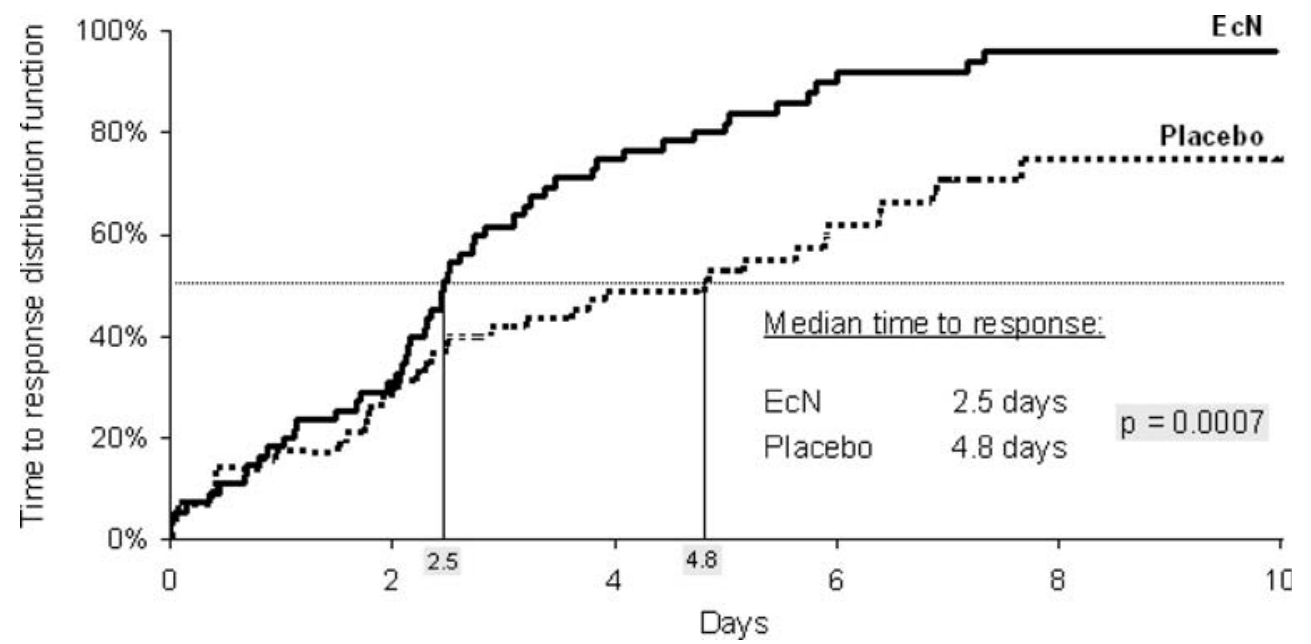

significantly superior to that of the placebo in terms of time to response ( $p=0.0007$; overall $p$ value of the group sequential test design) (Fig. 3). Analysis by centre showed no difference in the number of responders between treatment groups.

In total, diarrhoea was stopped in 52/55 patients (94.5\%) in the EcN group and $39 / 58$ patients $(67.2 \%)$ in the placebo group within 10 days. Fourteen patients dropped out of the trials (EcN, $n=1$; placebo, $n=13$ ) because of unsuccessful therapy. The diarrhoea did not cease within 10 days of treatment in two patients of the $\mathrm{EcN}$ and six patients of the placebo group.

\section{Secondary objectives}

An exploratory comparison showed a significant difference in the number of responders between the treatment groups $(p<0.0001$; ratio of rates: $1.406 ; 95 \% \mathrm{CI}: 1.162-1.701)$. A cumulative presentation of the number of responders on each study day showed a difference between $\mathrm{EcN}$ - and placebo-treated patients starting on day $3[\mathrm{EcN} 34 / 55$ (61.8\%) vs. placebo 24/58 (41.4\%); ratio of rates: 1.494 ; 95\%CI: 1.032-2.163] (Fig. 4). The difference increased until day $5[\mathrm{EcN} 45 / 55$ (81.8\%) vs. placebo $30 / 58$ (51.7\%); ratio of rates: $1.582 ; 95 \% \mathrm{CI}$ : $1.198-2.089]$ and then decreased slightly from day 6 to the end of study [EcN $52 / 55(94.5 \%)$ vs. placebo $39 / 58(67.2 \%)$; ratio of rates: 1.406; 95\%CI: $1.162-1.701)$.

Prior to the treatment regimen, almost no infant had a normal stool consistency. During the course of the study the patients of the EcN group showed a more pronounced improvement than their counterparts in the placebo group. The same trend was observed for the disappearance of abdominal pain $(28 / 30 \mathrm{EcN}$ patients vs. $24 / 33$ placebo patients) and abdominal cramps (17/18 EcN patients vs. 21/26 placebo patients) (Table 3 ).
Fig. 4 Response rates among the patients receiving the $\mathrm{EcN}$ solution $(n=55)$ and placebo $(n=58)$ during the course of the study

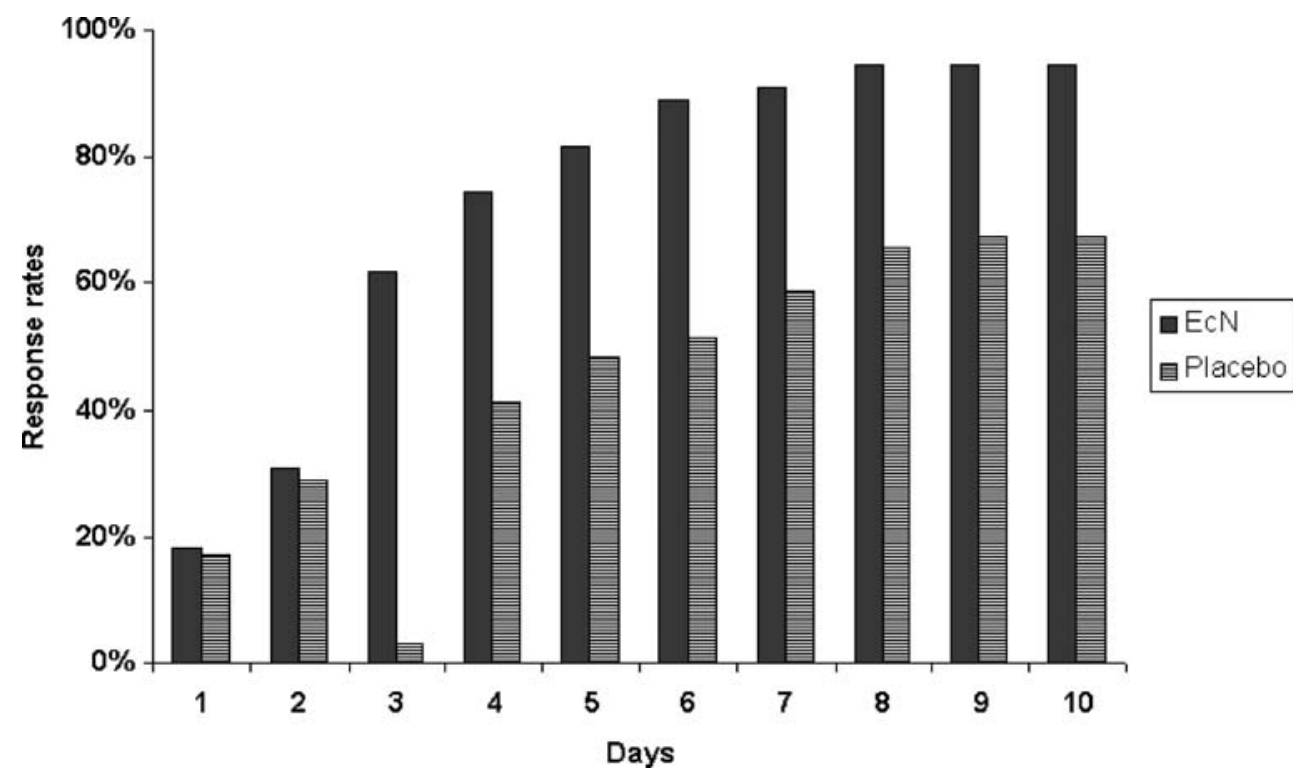


Table 3 Improvement of symptoms after treatment

\begin{tabular}{lll}
\hline & EcN (\%) & Placebo (\%) \\
\hline Normal stool consistency & 78.4 & 40.5 \\
No abdominal pain & 93.3 & 72.7 \\
No abdominal cramps & 94.4 & 80.8 \\
\hline
\end{tabular}

In addition, the general state of health, as assessed by the investigator during clinical examinations or by the parents by means of the diary, of the patients in the $\mathrm{EcN}$ group improved more clearly than that of the patients in the placebo group (data not shown). Body temperature showed an almost identical decrease over time in both treatment groups (EcN: $-0.5 \pm 0.4^{\circ} \mathrm{C}$; placebo: $-0.4 \pm 0.5^{\circ} \mathrm{C}$ ). The number of vomiting episodes was very small at baseline in all patients and decreased to $0 \%$ in both groups. In principle, body weight and the status of dehydration did not show any changes from baseline to the end of study in either treatment group. Only one patient in the placebo group experienced mild dehydration.

From baseline to study termination, pathogenic microorganisms disappeared in a similar number of patients in both treatment groups (14/27 patients in the EcN group and 12/21 patients in the placebo group). In the patients who were free from infectious agents at baseline, pathogenic microorganisms were detected at the end of study in $3 / 28$ and $6 / 37$ patients in the $\mathrm{EcN}$ and placebo group, respectively.

\section{Tolerance to treatment}

The study medication was well tolerated. Only $2 / 55$ patients $(3.6 \%)$ in the EcN group and $2 / 58$ patients $(3.4 \%)$ in the placebo group experienced one adverse event (AE) each. These were rhinitis and abdominal pain in the EcN-treated patients and two cases of acute otitis media in the patients receiving the placebo. According to the European Medicines Agency (EMEA) classification, none of these AEs are rated as "serious" or "severe" (regulatory guidance CPMP/ICH/ 377/95). The two placebo-treated patients with otitis media were removed from the study due to the AE being intolerable. For the two AEs in patients receiving the $\mathrm{EcN}$ treatment, complete recovery was documented. According to the parents, tolerance to treatment was slightly better in the EcN group than in the placebo group, whereas no notable difference was observed by the investigators (Table 4).

\section{Discussion}

The aim of this multicentre, prospective, confirmative, randomised, double-blind, placebo-controlled clinical trial of phase III was to investigate the therapeutic efficacy and safety of orally administered $\mathrm{EcN}$ in treating acute diarrhoea in infants and toddlers. The results showed that $\mathrm{EcN}$ was superior to the placebo in terms of both time to response and response rate. The difference in median duration of diarrhoea - 2.3 days - was statistically significant and also clinically important.

Acute diarrhoea in children is very often self-limiting within a few days. However, toddlers and young infants are in danger of developing dehydration and a deteriorating general health. Therefore, a fast-tracking antidiarrhoeal treatment would be beneficial. Several investigations have been carried out with probiotics for the treatment of acute gastroenteritis, and different meta-analyses and systematic reviews have been published in this field. All of these have demonstrated the efficacy of probiotics in treating or preventing diarrhoea. On average, the treatment of diarrhoea with lactobacilli, bifidobacteria and/or $S$. boulardii shortened the duration of diarrhoea by only $0.5-1.5$ days $[4,12,14,18,19]$.

Szajewska and Mrukowicz reviewed ten randomised, double-blind, placebo-controlled studies and concluded that the administration of probiotics led to a substantial reduction in the duration of acute diarrhoeal symptoms: an average of $20 \mathrm{~h}$ [18]. Moreover, a meta-analysis of nine clinical trials conducted by D'Souza et al. demonstrated that probiotics effectively prevented antibiotic-associated diarrhoea [4]. The work of van Niel et al. included nine randomised controlled studies with lactobacilli in acute infectious diarrhoea in children. In these studies, the duration of diarrhoea was significantly reduced by an average of 0.7 days along with the stool frequency [19]. Most recently, McFarland et al. examined the efficacy of probiotics in paediatric diarrhoea by analysing 39 randomised, controlled and blinded clinical trials comprising a total of 41 probiotic treatment arms [12]. Of these, $32(78 \%)$ reported efficacy. The latest meta-analysis of 39 trials by Sazawal et al. showed that probiotics prevented acute diarrhea, with a risk reduction among children off $57 \%$ (range: 35-71\%) [14]. Diarrhoea is one of the best-studied indications for probiotics, and treatment with EcN has been found to stop acute diarrhoea more rapidly than
Table 4 Tolerance to treatment in the two treatment groups

\footnotetext{
${ }^{\text {a }}$ Percentage is given in parenthesis
}

\begin{tabular}{|c|c|c|c|c|}
\hline & \multicolumn{2}{|c|}{ Assessed by parents } & \multicolumn{2}{|c|}{ Assessed by investigators } \\
\hline & $\mathrm{EcN}$ & Placebo & $\mathrm{EcN}$ & Placebo \\
\hline$\overline{\text { Very good }}$ & $11 / 55(20.0)^{\mathrm{a}}$ & $4 / 58(6.9)$ & $5 / 55(9.1)$ & $4 / 58(6.9)$ \\
\hline Good & $44 / 55(80.0)$ & $53 / 58(91.4)$ & $50 / 55(90.9)$ & $53 / 58(91.4)$ \\
\hline Poor & $0 / 55(0.0)$ & $1 / 58(1.7)$ & $0 / 55(0.0)$ & $1 / 58(1.7)$ \\
\hline
\end{tabular}


other probiotics. The efficacy of $\mathrm{EcN}$ was confirmed by a second multicentre, prospective, randomised, double-blind, placebo-controlled phase III study conducted by our group [7]. In that study, children with prolonged diarrhoea treated with EcN showed a more rapid onset of response to treatment than those treated with placebo (median: 2.4 vs. 5.7 days; $p<0.0001$ ). There was also a remarkable difference in the response rates, as determined on days 14 (EcN: 93.3\%; placebo: 65.8\%) and 21 (EcN: 98.7\%; placebo: $71.1 \%$ ), thus showing a statistically significant superiority of $\mathrm{EcN}$ on both days ( $p=0.0017$ and $p<0.001$, respectively).

In the present trial, high initial response rates in both groups represent the spontaneous healing known for acute gastroenteritis. The superiority of the $\mathrm{EcN}$ treatment became increasingly noticeable from 3 . The healing process was markedly faster in the EcN-treated patients than in the patients receiving placebo, a result which underlines the high efficacy of this probiotic.

The relatively high number of children with unspecific diarrhoea corresponds quite well to the frequent failure to detect the responsible pathogen in routine analyses. This is the reason why the results of this study are not helpful in answering the question whether $\mathrm{EcN}$ is more efficient in bacterial or viral diarrhea. This question should be addressed by future studies.

In the present study, EcN was safe and well-tolerated. There was no difference between the $\mathrm{EcN}$ and placebo treatments in terms of AEs, body weight, stool examinations and the assessment of tolerance. This result is in accordance with experience from clinical trials in premature and fullterm newborns where $\mathrm{EcN}$ was not only very safe but improved the microbial intestinal milieu of the treated infants and reduced the risk of acquiring pathogens early in life $[3,10,11]$. It has also been shown that prolonged colonisation with $\mathrm{EcN}$ protected infants at an age of 612 months from flatulence, diarrhoea or constipation when given immediately on the first 5 days after birth [16].

Our understanding of the effects of probiotics and their numerous modes of action has grown substantially in recent years. With regard to gastroenteritis, probiotics may improve symptoms by several mechanisms, including:

- competition with pathogens (for adherence to intestinal epithelium, for growth and survival in the gut) and inhibition of pathogen overgrowth;

- secretion of bacteriostatic/bactericidal peptides (e.g. colicins, microcins);

- enforcement of intestinal barrier function and reduction of microbial translocation;

- modulation of immune responses (local and/or systemic, e.g. stimulation of secretion of IgA by lymphocytes and defensins by enterocytes).
All of these mechanisms of action have been shown for E. coli strain Nissle 1917. The antagonistic activity of EcN against pathogens has been demonstrated in vitro in animal models and in humans $[1,10,13,17]$. In a pig model of intestinal infection, $\mathrm{EcN}$ was able to prevent acute secretory diarrhoea [15]. Among many other strain-specific characteristics $[2,5,6], \mathrm{EcN}$ exerts an intense immunomodulatory effect in children $[3,11]$. Here, $\mathrm{EcN}$ was found to stimulate the production of antibodies of mucosa-associated $\mathrm{B}$ lymphocytes and the systemic production of antibodies (IgM, IgA) in premature and fullterm children.

\section{Conclusion}

In summary, EcN showed a significant superiority to placebo in the treatment of acute diarrhoea in infants and toddlers. EcN treatment also improved the general state of health and its administration was safe and well tolerated.

\section{References}

1. Altenhoefer A, Oswald S, Sonnenborn U, Enders C, Schulze J, Hacker J, Oelschlaeger TA (2004) The probiotic Escherichia coli strain Nissle 1917 interferes with invasion of human intestinal epithelial cells by different enteroinvasive bacterial pathogens. FEMS Immunol Med Microbiol 40:223-229

2. Blum-Oehler G, Oswald S, Eiteljörge K, Sonnenborn U, Schulze J, Kruis W, Hacker J (2003) Development of strain-specific PCR reactions for the detection of the probiotic Escherichia coli strain Nissle 1917 in fecal samples. Res Microbiol 154:59-66

3. Cukrowska B, Lodinova-Zadnikova R, Enders C, Sonnenborn U, Schulze J, Tlaskalova-Hogenova H (2002) Specific proliferative and antibody response of premature infants to intestinal colonization with nonpathogenic probiotic E. coli strain Nissle 1917. Scand J Immunol 55:204-209

4. D'Souza AL, Rajkumar C, Cooke J, Bulpitt CJ (2002) Probiotics in prevention of antibiotic associated diarrhoea: meta-analysis. BMJ 324:1361-1366

5. Grozdanov L, Zähringer U, Blum-Oehler G, Brade L, Henne A, Knirel YA, Schombel U, Schulze J, Sonnenborn U, Gottschalk G, Hacker J, Rietschel ET, Dobrindt U (2002) A single nucleotide exchange in the wzy gene is responsible for the semirough $\mathrm{O} 6$ lipopolysaccharide phenotype and serum sensitivity of Escherichia coli strain Nissle 1917. J Bacteriol 184:5912-5925

6. Grozdanov L, Raasch C, Schulze J, Sonnenborn U, Gottschalk G, Hacker J, Dobrindt U (2004) Analysis of the genome structure of the nonpathogenic probiotic Escherichia coli strain Nissle 1917. J Bacteriol 186:5432-5441

7. Henker J, Blokhin BM, Bolbot YK, Maydannik VG, Joeres L, Wolff C, Schulze J (2006) Successful therapy of unspecific prolonged diarrhoea in infants and toddlers with the probiotic E. coli Nissle 1917. Gastroenterology 130:A-315

8. Kruis W, Fric P, Pokrotnieks J, Lukas M, Fixa B, Kascak M, Kamm MA, Weismueller J, Beglinger C, Stolte M, Wolff C, Schulze J (2004) Maintaining remission of ulcerative colitis with the probiotic $E$ coli Nissle 1917 is as effective as with standard mesalazine. Gut 53:1617-1623 
9. Lehmacher W, Wassmer G (1999) Adaptive sample size calculations in group sequential trials. Biometrics 55:1286-1290

10. Lodinova-Zadnikova R, Sonnenborn U (1997) Effect of preventive administration of a nonpathogenic Escherichia coli strain on the colonization of the intestine with microbial pathogens in newborn infants. Biol Neonate 71:224-232

11. Lodinova-Zadnikova R, Tlaskalova-Hogenova H, Sonnenborn U (1992) Local and serum antibody response in fullterm and premature infants after artificial colonization of the intestine with E. coli strain Nissle 1917 (Mutaflor ${ }^{\circledR}$ ). Pediatr Allergy Immunol $3: 43-48$

12. McFarland LV, Elmer GW, McFarland M (2006) Meta-analysis of probiotics for the prevention and treatment of acute pediatric diarrhea. Int J Probiotics Prebiotics 1:63-76

13. Patzer SI, Baquero MR, Bravo D, Moreno F, Hantke K (2003) The colicin $\mathrm{G}, \mathrm{H}$ and $\mathrm{X}$ determinants encode microcins $\mathrm{M}$ and H47, which might utilize the catecholate siderophore receptors FepA, Cir, Fiu and IroN. Microbiology 149:2557-2570

14. Sazawal S, Hiremath G, Dhingra U, Malik P, Deb S, Black RE (2006) Efficacy of probiotics in prevention of acute diarrhoea: a meta-analysis of masked, randomized, placebo-controlled trials. Lancet Infect Dis 6:374-382

15. Schroeder B, Duncker S, Barth S, Bauerfeind R, Gruber AD, Deppenmeier S, Breves G (2006) Preventive effects of the probiotic Escherichia coli strain Nissle 1917 on acute secretory diarrhea in a pig model of intestinal infection. Digest Dis Sci 51:724-731

16. Schröder H (1992) Entwicklung der aeroben Darmflora bei Neugeborenen nach Kolonisierung mit dem E.-coli-Stamm Nissle 1917. Kinderarzt 10:1619-1625

17. Schulze J, Lorenz A, Mandel L (1992) Colonization of Escherichia coli in different gnotobiotic animal models. Microbial Ecol Health Dis 5:4-5

18. Szajewska H, Mrukowicz JZ (2001) Probiotics in the treatment and prevention of acute infectious diarrhea in infants and children: a systematic review of published randomized, doubleblind, placebo-controlled trials. J Pediatr Gastroenterol Nutr 33: S17-S25

19. van Niel CW, Feudtner C, Garrison MM, Christakis DA (2002) Lactobacillus therapy for acute infectious diarrhea in children: a meta-analysis. Pediatrics 109:678-684 